\title{
Analysis of Circular Microstrip Antenna with Perturbation Segments
}

\author{
Talafimi FL.JIMOTO** Kazumasa TANAKA, Mitsuo TAGLCHI \\ Dept. of Flectrical Fng. \& Computer Science, Nagasaki Lniversity \\ 1-14 Bunkyo-machi, .Nagasaki-shi, 852-8131, Japan
}

\section{Introduction}

The cavity model is a simple and efficicnt analytical method on the microstrip antemen (MSA). In the cavity model the wall adnitianec at the aperture has to be determined in advance. The accuracy of the wall adrnittance influcnecs the input impedance and resonant frequency of antenna. Authors have proposed the formulation metlood on the wall admittance of arbitrarily shaped MSA and applicd it to the circular MSA [1], elliptical MSA[2] and rectangular MSA[3]. The wall admittance is defined in terms of the magnetic ficld due to the equivalent magnetic current on the aperture. The magnetic field is derived by using Green's functions in the spectral domain in order to consider the influence of diclectric substrate.

In this paper, the wall admittance of circular MSA with perturbation segments is calculated by our proposed method and the antenma is analyzed by the cavity model. Although the wall susceptance was formulated by the capacitance of fringe ficld around the aperture in previous studies[1][5], the suseptance given by our method consists of capacitance and inductance. The numerical results are presented which show that the susceptance of the primary mode in the neighborhood of the resonant frequency is inductive at the aperture of the perturbation segment and capacitive at the aperture except the perturbation segment.

\section{Theory}

Fig. 1 shows the circular MSA with perturbation segments and its coordinate. system. The dimensions of the two perturbation scgments are $b_{0} \leq r \leq a_{0} ; \phi=$ $-\phi_{1} \sim \phi_{1}$ and $\phi=\pi-\phi_{1} \sim \pi+\phi_{1}$, respectively. The antenna is excited at $\left(d_{0}, \phi_{0}\right)$ by a coaxial fecder through the diclectric substrate. The relative dielectric constant of substrate is $\varepsilon_{r}$.

The electromagnetic fields within and outside the cavity are denoted by $\boldsymbol{E}^{d}, \boldsymbol{H}^{d}$; $E^{e}, H^{e}$, respectively. The thickness of the substrate is assumed to be relatively small compared with the wavelength, so the electromagnetic fields within the cavity do not vary along the $z$ direction. The internal fields $E^{d}$ is expressed by the eigenfiunctions in the cylindrical coordinate system as follows,

$$
\begin{aligned}
& \text { In Region } 1 \\
& E^{d}=\sum_{n=1}^{N}\left(E_{z n}^{c . J}+E_{z \eta}^{s . J}\right) i_{i} \\
& E_{z n}^{c . I}=A_{n} J_{n}\left(k_{1} r\right) \cos (n \phi) \quad, \quad E_{i n}^{z, J}=B_{n} J_{n}\left(k_{1} r\right) \sin (n \phi)
\end{aligned}
$$

In Region 2

$$
E^{d}=\sum_{n=1}^{N}\left(E_{z=}^{c J}+E_{z n}^{s J}+E_{z n}^{c N}+E_{z n}^{s \Lambda}\right) i_{z}
$$




$$
\begin{array}{lll}
E_{z n}^{c J}=C_{n} J_{n}\left(k_{11} r\right) \cos (n \phi) \quad, \quad & E_{z n}^{c N}=D_{n} N_{n}\left(k_{1} r\right) \cos (n \phi) \\
E_{z n}^{s J}=E_{n} J_{n}\left(k_{1} r\right) \sin (n \phi) & , \quad E_{z n}^{s N}=F_{n} N_{n}\left(k_{1} r\right) \sin (n \phi)
\end{array}
$$

where $J_{n}\left(k_{1} r\right)$ and $N_{n}\left(k_{1} r\right)$ are Bessel and Neumann functions of order $n$, respectively. $\left\{A_{n}\right\}-\left\{F_{n}^{\prime}\right\}$ are $11 n k n o w n$ coefficients to be determined from the continuity conditions of the electromagnetic fields between the region 1 and 2 and the impedance boundary condition at the aperture[6]. Since the radiations from the aperture 2 and 4,6 and 8 are cancelled each other, the magnetic currents on these apertures don't contribute to the radiation field. Therefore, the impedance boundary condition is applied as follows:

$$
\int_{S_{1}+S_{3}+S_{5}+S_{T}}\left\{\sum_{n=1}^{N}\left(y_{n}^{c J} E_{z n}^{c J}+y_{n}^{c N} E_{z n}^{c N}+y_{n}^{s J} E_{z n}^{s J}+y_{n}^{s N} E_{z n}^{s N}\right)+H_{\dot{c}}^{d}\right\} d S^{\prime}=0 .
$$

The wall admittances $y_{n}^{c J}, y_{n}^{c N}, y_{n}^{s J}$ and $y_{n}^{s N}$ are defined by the magnetic fields

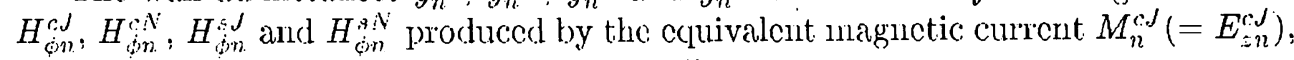
$M_{n}^{c . N}\left(=E_{z n}^{s N}\right), M_{n}^{s J}\left(=E_{z n}^{s, J}\right)$ and $M_{n}^{s N}\left(=E_{z n}^{s N}\right)$ on the aperture, respectively.

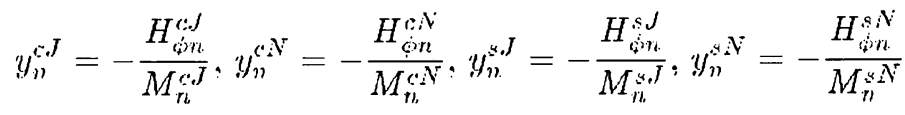

The magnetic ficlds $\boldsymbol{H}^{e}$ at any point on the apcrture produced by the magnetic: current $M\left(=M i_{\omega}\right)$ are expressed as the following equation[1]-[3],

$$
\begin{aligned}
H^{e}=-j \omega \int_{S_{1}+S_{3}+S_{3}+S_{7}} & \left(i_{X} G_{F}^{X X}+i_{Y} G_{F}^{Y X}\right) M d S^{\prime} \\
& +\frac{1}{j \omega} \int_{S_{1}+S_{3}+S_{5}+S_{7}}\left(\nabla G_{V}\right)\left(\nabla^{\prime} \cdot M\right) d S^{\prime}
\end{aligned}
$$

where for the reason mentioncd above, the magnetic currents on the apcrture 2,4 , 6 and 8 are neglected. Crecen's functions $G_{r^{\prime}}^{X}, G_{r}^{Y}{ }^{X}$ and $G_{V^{\prime}}$ in the spatial domain are oblained by applying the inverse Fourier translorm to Green's functions in the spectral domain [1]-[3].

\section{Results and Discussion}

Fig. 2 shows the calctulated wall admittances $y_{1}^{\mathrm{c} J}$ and $y_{1}^{\mathrm{s} J}$ of the primary mode at the resonant frecuency. In this paper, the disctussions on $y_{1}^{C^{N}}$ and $y_{1}^{S_{N}}$ are omitted because $y_{1}^{N^{N}}$ and $y_{1}^{S^{N}}$ have the same characteristics as $y_{1}^{J J}$ and $y_{1}^{S J}$. The calculated wall conductances $g_{1}^{\leftarrow J}$ and $g_{1}^{s J}$ are constant in the aperture 1 and 3 , respectivcly.

Substituting eqn.(8) into eqn.(7), the wall susceptance $b_{n}^{\text {c.J }}$ is expressed as follows,

$$
\begin{aligned}
& b_{n}^{c J}=j \omega C_{n}-\frac{j}{\omega L_{n}}
\end{aligned}
$$

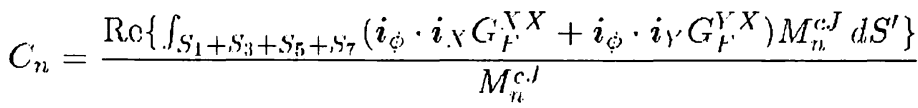

$$
\begin{aligned}
& L_{n}=\frac{M_{n}^{(J)}}{\operatorname{Re}\left\{\int_{S_{1}+S_{3}+S_{5}+S_{\tau}}\left(i_{\varphi} \cdot \nabla G_{V^{\prime}}^{\prime}\right)\left(\nabla^{\prime} \cdot i_{\varphi} M_{n}^{C^{J} J}\right) d S^{\prime}\right\}} .
\end{aligned}
$$

$b_{n}^{s . J}$ is given by replacing $M_{n}^{c . J}$ in equs.(9)-(11) by $M_{n}^{s J}$. Equs.(9)-(11) indicate that the wall susceptance is equivalent to LC parallel circuit and $\mathrm{C}$ and $\mathrm{L}$ comrespond to 
vector and scalar potcentials, respectively. As shown in Fig. 2, the wall susceptance $b_{1}^{c . J}$ is capacitive at the apertures 1 and $3 . b_{1}^{s . J}$ is inductive at the aperture 1 and capacitive at the aperture 3.

Fig. 3 shows the frequency characteristics of $b_{1}^{c J}$ at $\phi=0^{\circ}$ and $b_{1}^{\text {s.J }}$ at $\phi=90^{\circ}$, which influence the elcetromagnetic ficlds within the antenna cavity. $b_{1}^{\mathrm{cJ}}$ and $b_{1}^{\mathrm{sj}}$ vary from inductive to capacitive as the frequency becomes higher.

Fig. 4 shows the frefuency charactcristics of calculated input impedances of circular MSA with perturbation segments. The resonant frequencies are $6.59 \mathrm{GH} \%$ and the minimum axial ratio is $0.07 \mathrm{~dB}$ at $6.554 \mathrm{GHz}$.

\section{Conclusion}

The wall admittance of circular MSA with perturbation segments is evaluated by using Green's functions in the spectral domain. The wall susceptances can be regarded as the LC parallel circuit. In neighborhood of the resonant frequency, the wall susceptance of the primary mode is inductive at the aperture of the perturbation segment and is capacitive at the apertine except the perturbation segment.

The input impedances and axial ratios are calculated by the cavity model with this wall admittances and the geometry of the patch to radiate circular polarization is detcrmined. The comparison between the numerical and the measured ones will be reported at the Symposiunn sitc.

\section{Acknowledgment}

This research was supported in part by Grant-in-Aid for Scicntific Research 09750429 from the Ministry of Education, Scicnce and Culture of Japan.

\section{References}

[1] 'T.Fujimoto, M.'laguchi and K.'l'anaka : "Surface arlmittance of circular microstrip antemin", Proc. IEEE, AP-S, Im.t.,Symp., vol.1, pp.696-699, Junc 1995.

[2] T.Fujimoto, M.Taguchi and K.Tanalia : "Surface admittance of elliptical microstrip antemin", Proce of Int., Symp. on. Antennas and Propagat., vol. 2, pp.541-544, Scp. 1996.

[3] T Mizuguchi, T.Fujimoto, K.Tanalar and M.Taguchi : "Surface: admittance of rectangular microsirip antema", Pros: Techanical reporl of IEICE, vol. AP96-128, pp.65-70, Janl. 1997.

[4] L.C.Shen, S.A.Long, M.R.Allerding and M.D.Walton : "Resonant frequency of a circular clisc, printed-circuit antemna", IEEE Trans., Antennas Propagat.,, vol. AP-2.5. pp.595-596, July 197 i .

[5] W.C.Chew and J.A.Iiong : "Effects of fringing fields on the capacitance of circular microstrip disk", IEEE Trans., Microwave Theory Tech.e, vol. MTT-28, no.2, pp.98103, Feb. 1980.

[6] L.C.Shen : "Analysis of circular-disc: printed-circuit antenna", Proc. IEE, vol. 126, 110.12. pp.122()-1222, Dec. 1979. 


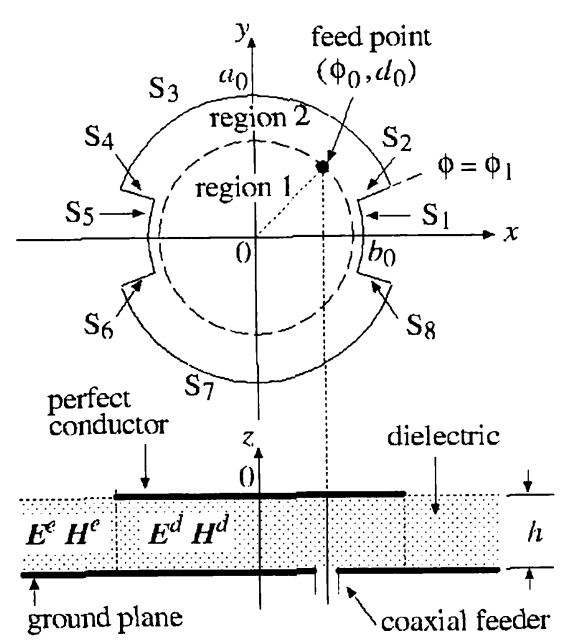

Fig. 1 Circular MSA with perturbation segments

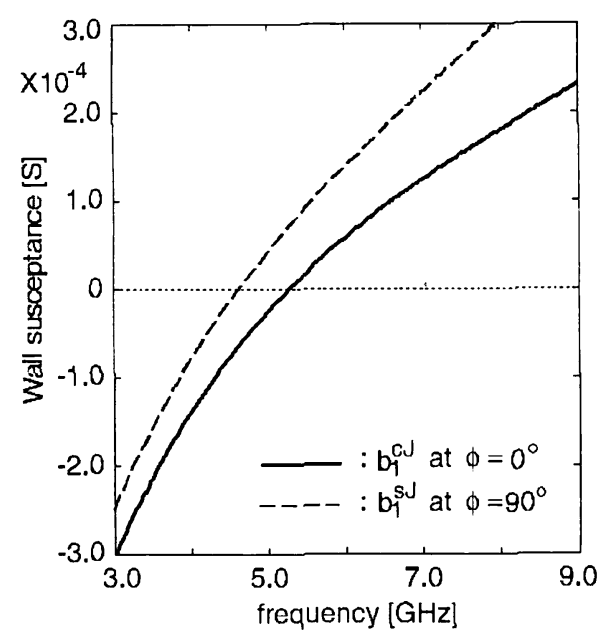

Fig. 3 Frecucucy charactcristics of calculated wall susceptances

$a_{0}=9.0 \mathrm{nrmm}, b_{0}=6.7 \mathrm{mmm}, \quad l_{l}=0.764 \mathrm{mmm}$, $\varepsilon_{r}=2.15, \phi_{1}=4^{\circ}$

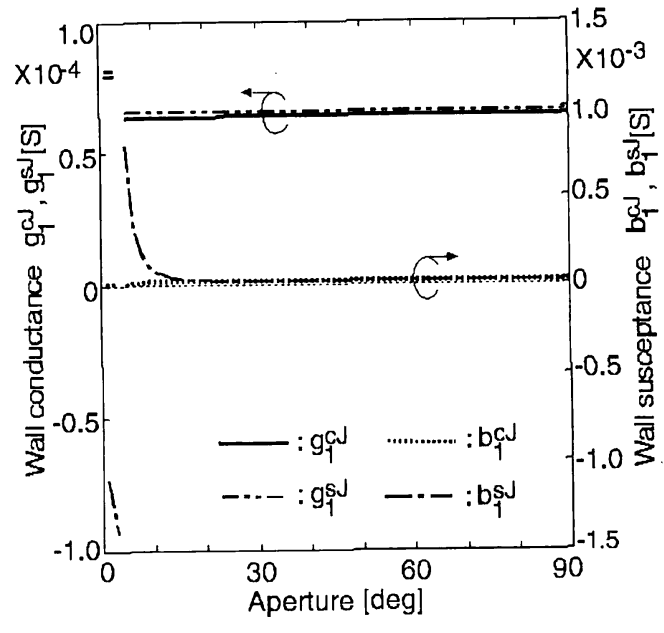

Fig. 2 Calculated wall admittances distribution at apertures

$a_{0}=9.0 \mathrm{~mm}, b_{0}=6.7 \mathrm{~mm}, h_{1}=0.764 \mathrm{~mm}$, $\varepsilon_{r}=2.15, \phi_{1}=4^{\circ}$, frequency $=6.59 \mathrm{GHz}$

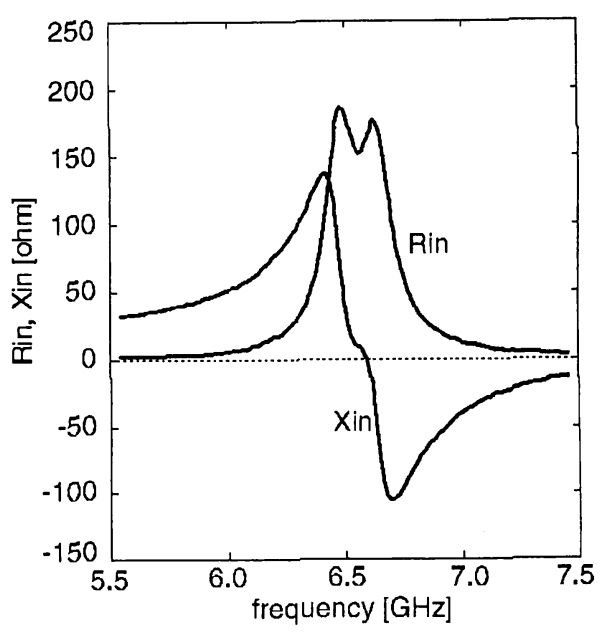

Fig. 4 Frcqucncy charactcristics of calculated input impedance

$a_{0}=9.0 \mathrm{mmm}, b_{0}=6.7 \mathrm{mmn}, h_{t}=0.764 \mathrm{~mm}$, $c_{r}=2.15, \phi_{1}=4^{\circ}, d_{0}=6.0 \mathrm{~mm}, \phi_{0}=45^{\circ}$; $N=5$ 